\title{
Middle East Respiratory Syndrome Coronavirus-Encoded ORF8b Inhibits RIG-I-Like Receptors by a Differential Mechanism
}

\author{
Jeong Yoon Lee ${ }^{1}$, Seong-Jun $\mathrm{Kim}^{2}$, and Jinjong Myoung ${ }^{1 *}$ \\ ${ }^{1}$ Korea Zoonosis Research Institute, Department of Bioactive Material Science and Genetic Engineering Research Institute, Chonbuk National \\ University, Jeonju 54531, Republic of Korea \\ ${ }^{2}$ Center for Convergent Research of Emerging Virus Infection, Korea Research Institute of Chemical Technology, Daejeon 34114, Republic of Korea
}

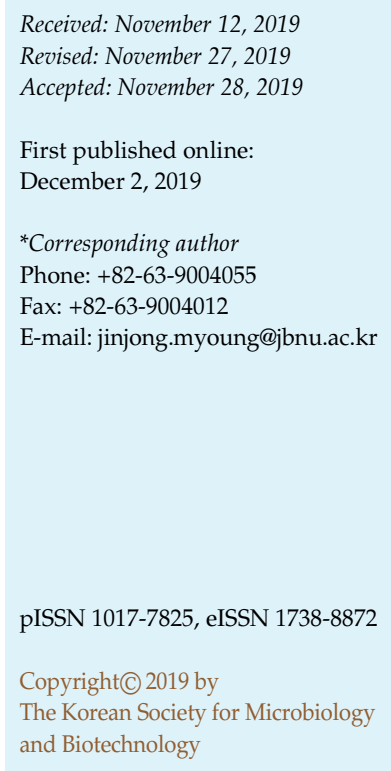

\begin{abstract}
Middle East respiratory syndrome coronavirus (MERS-CoV) belongs to the genus Betacoronavirus and causes severe morbidity and mortality in humans especially when infected patients have underlying diseases such as chronic obstructive pulmonary disease (COPD). Previously, we demonstrated that MERS-CoV-encoded ORF8b strongly inhibits MDA5- and RIG-I-mediated induction of the interferon beta (IFN- $\beta$ ) promoter activities. Here, we report that ORF8b seemed to regulate MDA5 or RIG-I differentially as protein levels of MDA5 were significantly down-regulated while those of RIG-I were largely unperturbed. In addition, ORF8b seemed to efficiently suppress phosphorylation of IRF3 at the residues of 386 and 396 in cells transfected with RIG-I while total endogenous levels of IRF3 remained largely unchanged. Furthermore, ORF8b was able to inhibit all forms of RIG-I; full-length, RIG-I-1-734, and RIG-I-1-228, the last of which contains only the CARD domains. Taken together, it is tempting to postulate that ORF8b may interfere with the CARD-CARD interactions between RIG-I and MAVS. Further detailed analysis is required to delineate the mechanisms of how ORF8b inhibits the MDA5/ RIG-I receptor signaling pathway.
\end{abstract}

Keywords: MERS-CoV, interferon, RIG-I

\section{Introduction}

Middle East respiratory syndrome coronavirus (MERS$\mathrm{CoV})$ was first identified by Zaki et al. [1, 2] in its association with acute pneumonia in a 60-year-old man in Saudi Arabia. The novel virus, termed "human coronavirus Erasmus Medical Center" (hCoV-EMC), was isolated from the sputum of the patient and sequence analysis of its genome displayed close similarity to bat coronaviruses HKU4 and HKU5. Subsequent viral genome analysis revealed that MERS-CoV belongs to the family Coronaviridae of the order Nidovirales [3,4]. Among four genera of the Coronaviridae, the virus is classified as lineage $C$ of the $\beta$ coronaviruses $[1,3]$. Approximately $80 \%$ of all MERS-CoV human infection cases have been reported in the Arabian Peninsula [5-7], which is explainable by the differential distribution of camel species. It is now well established that MERS-CoV is a fatal zoonotic pathogen from dromedary, but not bactrian, camels to humans $[4,8-$ 10]. In fact, a number of coronaviruses that infect humans and livestock have been shown to evolve from bat coronaviruses, including MERS-CoV [4,11-14], severe acute respiratory syndrome coronavirus (SARS-CoV) [1517], swine acute diarrhea syndrome coronavirus (SADSCoV) [18], and porcine epidemic diarrhea virus (PEDV) [19]. In addition, over a thousand coronavirus sequences were identified and reported from bats [20, 21]. It seems that bats are the major coronavirus reservoir and as such it is likely that they will continue to serve as the brewing pots for new deadly coronaviruses [22].

Upon virus infection, type I interferons (IFN) are rapidly induced by sequential activation of signaling molecules [23-27]. Upon RNA virus infection, viral RNA genomes are recognized by intracellular RNA sensors: melanoma differentiation-associated protein 5 (MDA5) and retinoic acid inducible gene 1 (RIG-I) [28-30]. As such, MDA5 and 
RIG-I are called pattern recognition receptors (PRRs). Binding of cognate ligands with MDA5 or RIG-I induces their conformational changes, resulting in the interaction of caspase activation and recruitment domains (CARDs) with those of mitochondrial antiviral signaling adaptor protein (MAVS), also known as interferon- $\beta$ promoter stimulator 1 (IPS-1). MAVS protein in turn undergoes conformational changes polymerizing on the membrane of mitochondria [31-35], which subsequently phosphorylates and activates downstream signaling molecules: I kappa B kinase epsilon $(\mathrm{IKK} \varepsilon$ ) and TANK-binding kinase 1 (TBK1) [34, 36-40]. Activation of IKK $\varepsilon$ and TBK1 induces nuclear translocation of transcription factors which are critical for the activation of the interferon $\beta$ promoter: interferon regulatory factor 3 (IRF3) [41-43] and nuclear factor kappa-light-chainenhancer of activated B cells (NF-кB) [22, 44, 45], inducing the expression of type I interferons and subsequently interferon-stimulated genes (ISGs). These gene products play an important role against invading viral pathogens in an auto- and paracrine manner [46-50].

Previously, we demonstrated that MERS-CoV-encoded accessory proteins are involved in evasion of the host IFN responses [22, 46]. Especially, ORF8b was identified as a novel antagonist of both IFN- $\beta$ [46] and NF- $\kappa B$ [22] activation. Interestingly, ORF8b-mediated inhibition was via interference of intracellular pattern recognition receptors (MDA5 and RIG-I) as well as TBK1. In addition, ORF4a and ORF4b also blocked induction of IFN- $\beta$ and NF- $\kappa B$ either individually or in combination.

Ectopic expression of RLR CARD can induce polymerization of MAVS, functioning as a constitutively active variant of RIG-I-like receptors (RLRs). In this study, we took advantage of a constitutively active RIG-I (RIG-I-1-228), which contains only two N-terminal CARDs. Interestingly, ORF8b inhibited both wild-type and constitutively active RIG-I's, suggesting that ORF8b may prevent efficient CARD-CARD interactions between RIG-I and MAVS. Further studies will shed light on the exact mechanisms of ORF8b-mediated inhibition of IFN signaling in cells. Taken together, we demonstrated that ORF8b efficiently inhibits RIG-I-mediated IFN- $\beta$ promoter activation, possibly through the interaction with the N-terminal CARD domains.

\section{Materials and Methods}

\section{Cell Culture and Reagents}

Human embryonic kidney 293T (HEK293T) cells were purchased from the American Type Culture Collection (ATCC, USA) and maintained in Dulbecco's Modified Eagle's Medium DMEM
(High-glucose, Welgene, Korea) supplemented with 10\% fetal bovine serum (FBS, Welgene) and 1\% penicillin/streptomycin (Thermo Fisher Sicentific, USA) [51, 52]. Cells were incubated at $37^{\circ} \mathrm{C}$ in a humidifying $5 \% \mathrm{CO}_{2}$ incubator $[35,53,54]$. Restriction enzymes for cloning were purchased from either Enzynomics (Korea) or New England Biolabs (NEB, USA). Firefly luciferase and Beta-Glo Assay System for luciferase assay [34] were procured from Promega (USA). Anti-FLAG antibody (M2) was purchased from Millipore Sigma (USA) and used at 1:5000 dilution. The following antibodies were obtained from Cell Signaling (USA): HA tag-specific mouse monoclonal antibody (6E2, 1/1000), horseradish peroxidase (HRP)-conjugated rabbit monoclonal anti-GAPDH antibody (14C10, 1/2000), HRP-conjugated anti-mouse IgG antibody (1/2000).

\section{DNA Constructs}

Construction of expression plasmids for accessory genes of MERS-CoV and the host signaling molecules (RIG-I and IRF3) was described elsewhere [22, 46]. RIG-I-1-228, consisting only of two N-terminal CARDs, and RIG-I-1-734, deficient of C-terminal regulatory domain (RD), were generated by $\mathrm{PCR}$ amplification and cloning into pcDNA3.1-Hygro-JY4-HAN-GS3 [55]. An IRF3-1390-expressing plasmid was similarly generated. IRF3-5D (S396D, S398D, S402D, T404D, and S405D), a constitutively active IRF3, was constructed by replacing the indicated Ser/Thr residues with phosphomimetic Asp (D).

\section{Transfection and Luciferase Reporter Assay}

$4 \times 10^{5}$ HEK293T cells were seeded in a 6-well plate at $24 \mathrm{~h}$ before transfection and a mixture of plasmids complexed with polyethylenimine (PEI, Sigma-Aldrich, USA) were transfected as described before [23, 27, 35]: $500 \mathrm{ng}$ IFN- $\beta$-luc, $100 \mathrm{ng} \beta$-gal, $1,000 \mathrm{ng}$ individual accessory gene of MERS-CoV, and $500 \mathrm{ng}$ each signaling molecule (RIG-I, IRF3, etc.) as indicated.

\section{Western Blotting}

Protein quantification was performed using Pierce BCA Protein Assay Kit (Thermo Fisher Scientific) as described in the manufacturer's instructions. 4X Laemmli Sample Buffer (Bio-Rad, USA) and 2-Mercatoethanol (Bio-Rad) were mixed at 9:1 ratio, and then the mixture and $15 \mu \mathrm{g}$ of sample were mixed at 1:4 ratio. After incubating at $100^{\circ} \mathrm{C}$ for $5 \mathrm{~min}$, the samples were separated on a SDS-PAGE gel. The samples were transferred on a NC blotting membrane (GE Healthcare Life Sciences, USA) at $100 \mathrm{~V}$ for $120 \mathrm{~min}$. The membrane was blocked by $5 \%$ skim milk (BD, USA) and then the primary antibodies at $4^{\circ} \mathrm{C}$ for $\mathrm{O} / \mathrm{N}$ : A mouse monoclonal anti-FLAG antibody (M2) (1:5000) from Millipore Sigma; HA-tagged (6E2) mouse monoclonal antibody (1:1000) from Cell Signaling; IRF-3 antibody (SL-12) for total IRF-3 form (1:1000) from Santa Cruz; Recombinant Anti-IRF3 (phospho S386) antibody (1:1000) from abcam; Phospho-IRF-3 (Ser396)(4D4G) Rabbit mAb (1:1000) from Cell Signaling; horseradish peroxidase (HRP)-conjugated rabbit monoclonal anti-GAPDH (14C10) (1:2000) 
from Cell Signaling. The secondary antibodies were incubated at RT for 60 min: horseradish peroxidase (HRP)-conjugated rabbit monoclonal anti-GAPDH (14C10) (1:2000), HRP-conjugated antirabbit IgG antibody (1:2000) from Cell Signaling. Amersham ECL Prime Western Blotting Detection Reagent (GE Healthcare Life Sciences) for $\alpha$-FLAG, $\alpha$-mouse, $\alpha$-rabbit, and Amersham ECL Western Blotting Detection Reagent (GE Healthcare Life Sciences) for $\alpha$-HA and $\alpha$-GAPDH were treated on the membrane at RT for $1 \mathrm{~min}$.

\section{Statistical Analysis}

Paired two-tailed Student's $t$-test was performed for statistical analysis. $P$-value $<0.05$ was considered as significant difference.

\section{Results}

\section{ORF8b-Mediated Inhibition of RLRs Led to Lower Levels of IRF3 Phosphorylation}

Previously, we demonstrated that ORF8b strongly inhibits MDA5- and RIG-I-mediated induction of the IFN- $\beta$ signaling pathway $[22,46]$. Interestingly, mechanisms of ORF8b-mediated inhibition seem to vary as protein levels of MDA5 were down-regulated by ORF8b while those of RIG-I were barely affected. To further investigate if endogenous levels of phosphorylation and activation of IRF3, the key regulator of the IFN- $\beta$ signaling pathway, are differentially affected by ORF8b, HEK 293T cells were cotransfected with ORF8b and MDA5 (Fig. 1A) or RIG-I (Fig. 1B). As expected, protein levels of MDA5 were significantly down-regulated by co-expression of ORF8b. As previously shown, ORF8b significantly inhibited RIG-Imediated induction of the IFN- $\beta$ signaling although protein levels of RIG-I were not affected by ORF8b. Furthermore, while total endogenous protein levels of IRF3 were comparable, levels of phospho-IRF3 at the residues 386 and 396 were significantly lower when ORF8b was coexpressed. These data suggest that ORF8b may differentially inhibit MDA5- or RIG-I-mediated induction of the IFN- $\beta$ pathway.
A

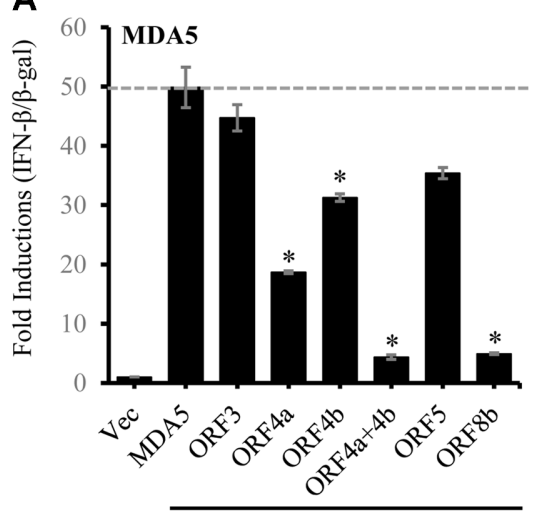

HAN-GS3-MDA5

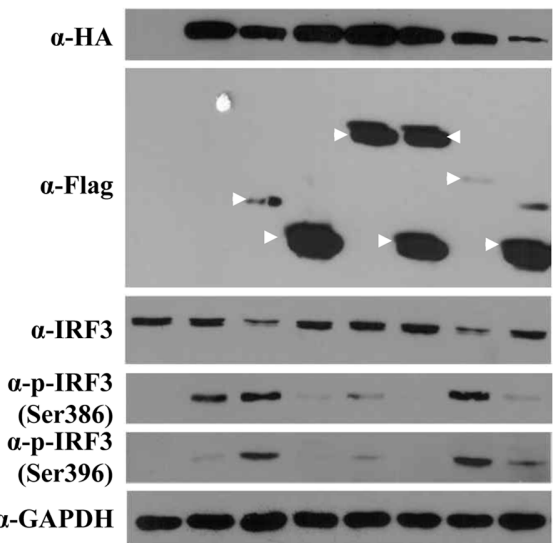

B

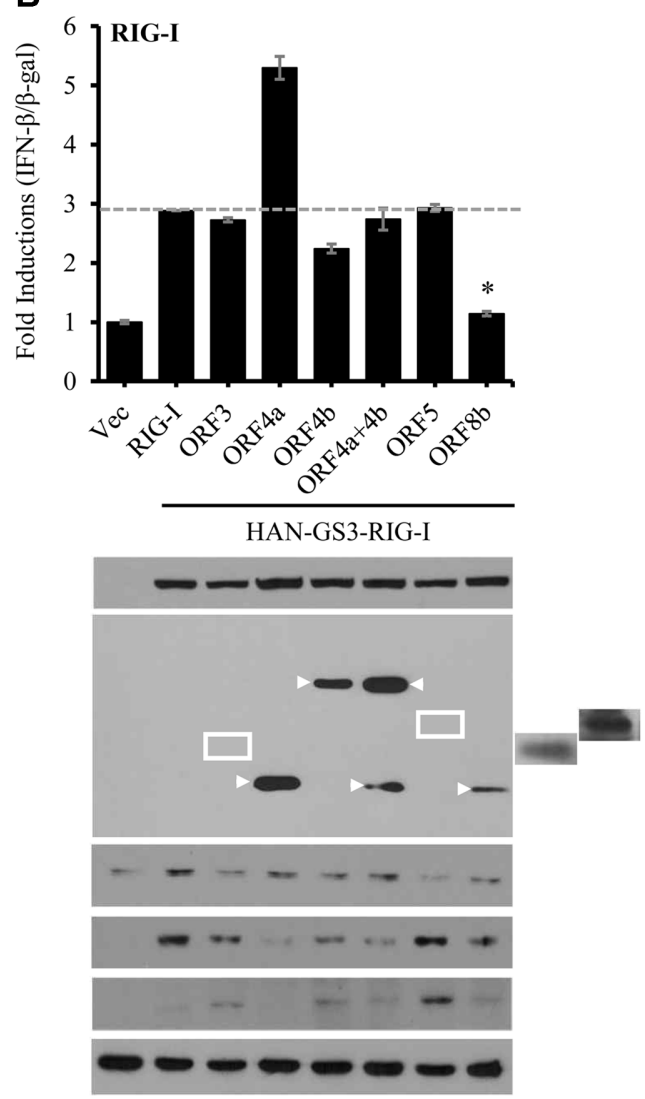

Fig. 1. ORF8b inhibits phosphorylation of IRF3 induced by RLRs.

HEK293 cells were transfected with MDA5 (A) or RIG-I (B) together with each individual MERS-CoV accessory gene. At $24 \mathrm{~h}$ post-transfection, cells were harvested for luciferase assay (top panels) and western blotting (bottom panels). Note that RIG-I's were HA-tagged at the N-terminus with a spacer (3X GGGGS). All MERS-CoV accessory proteins are tagged with the FLAG tag at the N-terminus. 
A

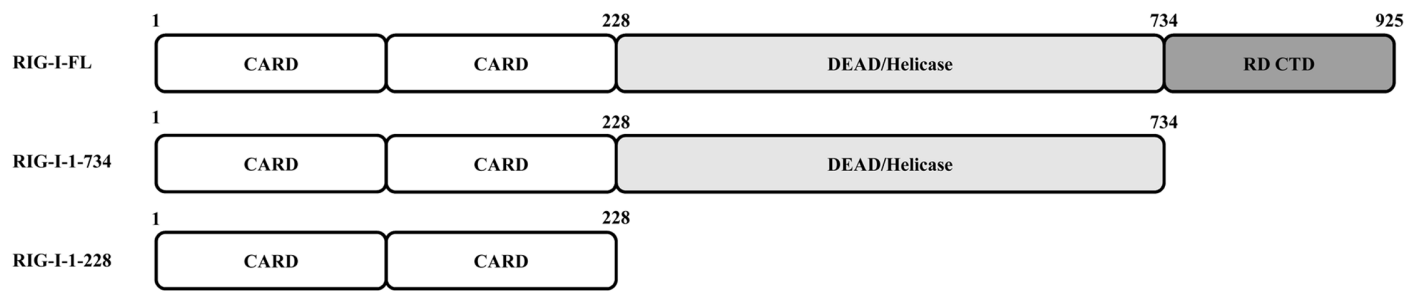

B

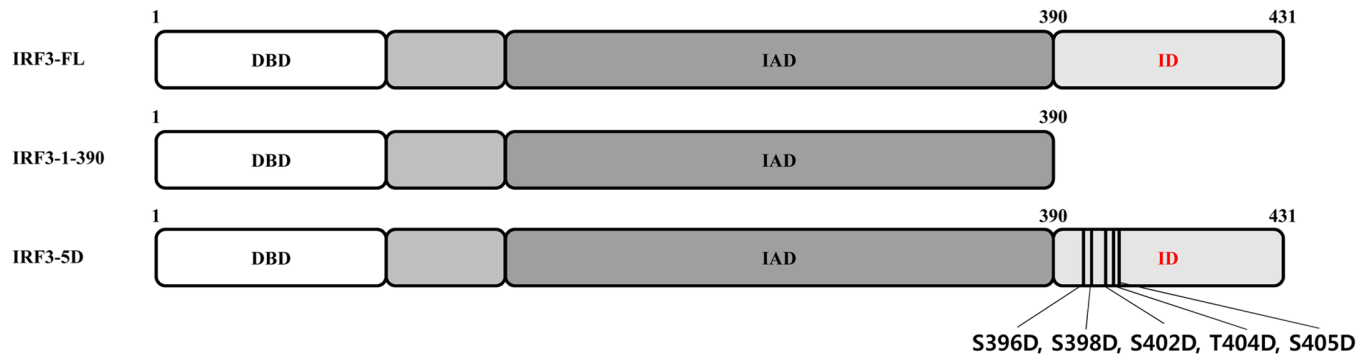

Fig. 2. Schematic diagram of variants of RIG-I and IRF3.

Each indicated region of RIG-I and IRF3 was PCR amplified and cloned into an expression vector. Their expression was confirmed by western blotting before functional assays.

\section{ORF8b Inhibited the CARD-Domain-Only Constitutively}

\section{Active RIG-I}

As protein levels of RIG-I were not affected by ORF8b, we hypothesized that ORF8b may inhibit the IFN- $\beta$ signaling pathway by interfering with protein-protein interactions, which are critical for the optimal activation of the pathway. For example, the CARD domain of RIG-I interacts with the CARD domain of MAVS, inducing its
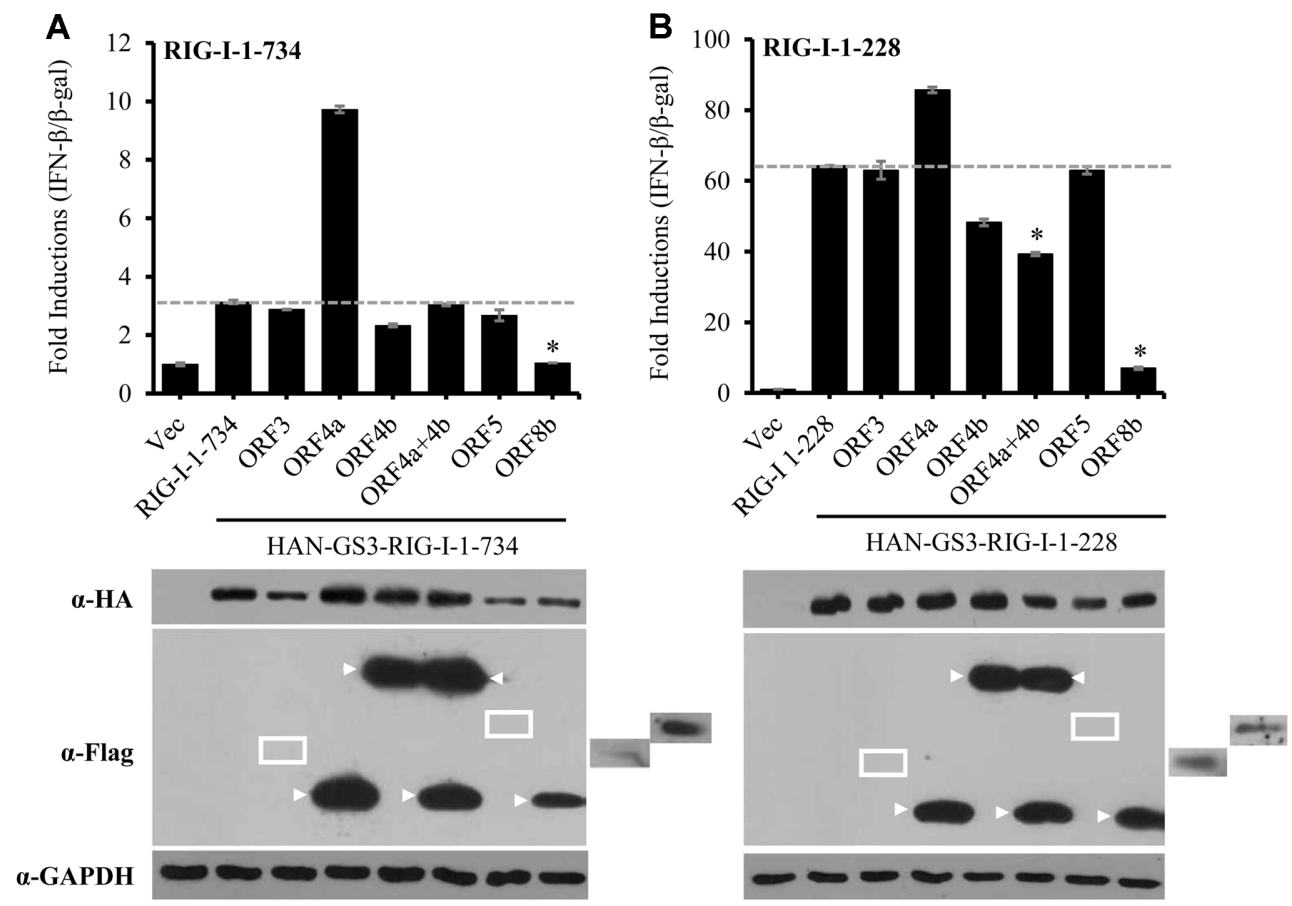

Fig. 3. ORF8b inhibits a CARD-domain-only constitutively active RIG-I.

HEK293 cells were transfected with RIG-I-1-734 (A) or RIG-I-1-228 (B) together with each individual MERS-CoV accessory gene. Subsequently, luciferase assay and western blotting was performed as described in Fig. 1. 
polymerization and activation. Therefore, we employed a constitutively active RIG-I (Fig. 2A) that contains only the CARD domain. In addition, we also took advantage of RIG-I-1-734, which lacks the C-terminal regulatory domain. As demonstrated in Fig. 3, ORF8b strongly inhibited both RIG-I-1-734- and RIG-I-1-228-mediated activation of the IFN- $\beta$ promoter activities. These data suggest that ORF8b may inhibit CARD-CARD interactions between RIG-I and MAVS.

\section{ORF8b Did Not Inhibit IRF3 Directly}

As IRF3 plays the key role in the activation of the IFN- $\beta$ promoter, we tested whether activation of IRF3 was regulated by ORF8b. As depicted in Fig. 2B, 3 forms of IRF3 constructs were generated and utilized: full-length, IRF3-1390 (deficient of the C-terminal regulatory domain), and constitutively active IRF3-5D, which contains five substitutions of serine and threonine with aspartate in the regulatory domain in the $\mathrm{C}$-terminus as indicated (Fig. 2B). As shown in Fig. 4, none of MERS-CoV-encoded accessory protein seemed to inhibit any forms of IRF3. These data suggest that ORF8b-mediated inhibition of the IFN- $\beta$ signaling pathway does not involve direct inhibition of IRF3. Rather, it is likely that the target(s) of ORF8b is upstream of it.

\section{Discussion}

In this study, we demonstrated that MERS-CoV-encoded $\mathrm{ORF} 8 \mathrm{~b}$ is a strong antagonist of RIG-I-mediated activation of IFN- $\beta$ signaling. Genomes of invading viruses are recognized by two intracellular RNA sensors: MDA5 and RIG-I. Although both MDA5 and RIG-I recognize and bind to the non-self RNA molecules, the type and nature of RNA ligands seem to vary $[34,56,57]$ : MDA5 binds to relatively long RNAs without particular restrictions on the RNA structure while RIG-I typically recognizes short blunted 5'triphosphated double- or single-stranded RNAs. RIG-I is inactive in the cytosol with the C-terminal regulatory domain (RD) blocking the CARD domains. Once RIG-I binds to its cognate ligand via its helicase domain, it undergoes conformational changes to release CARD domains. RIG-I CARD domains in turn interact with the CARD domain of MAVS protein on the outer membrane of the mitochondria, inducing its polymerization. As the CARD domain of RIG-I is a protein interaction motif for the formation of larger protein complexes, the inhibition of RIG-I-228 by MERS-CoV ORF8b strongly suggests that ORF8b prevents RIG-I interactions with MAVS (Fig. 3). This notion is supported by the unperturbed levels of RIG-I protein by ORF8b (Fig. 1B). This is in sharp contrast to
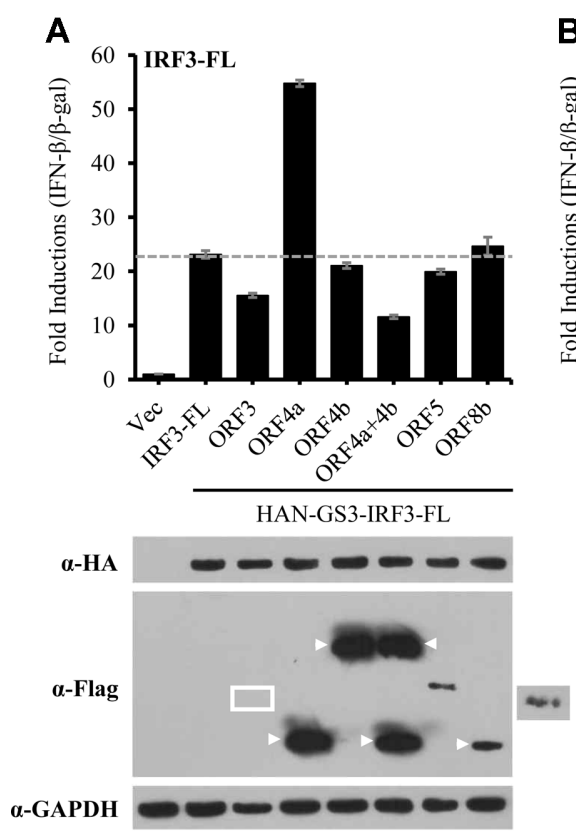
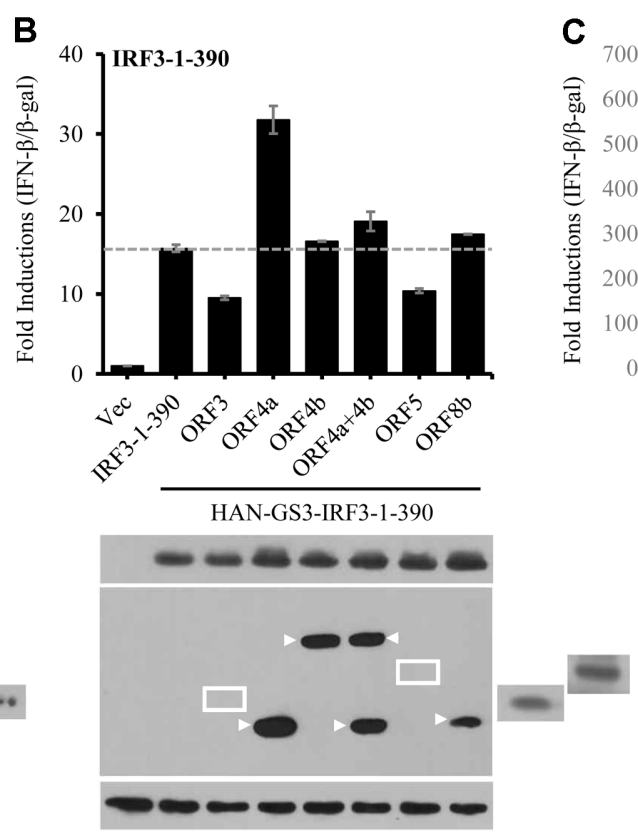
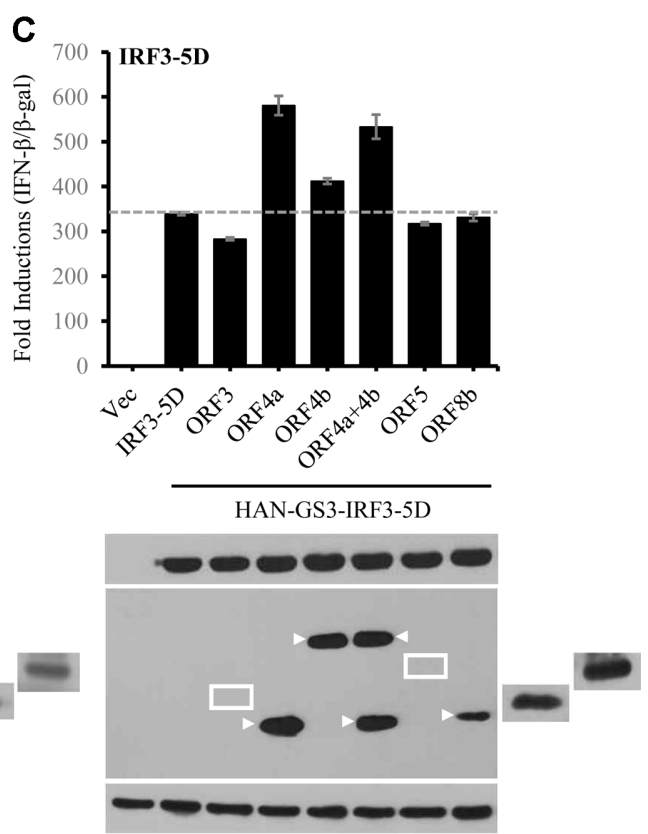

Fig. 4. ORF8b did not suppress IRF3-mediated activation of IFN- $\beta$ signaling. HEK293 cells were transfected with IRF3 (A), IRF3-1-390, or IRF3-5D (C) together with each individual MERS-CoV accessory protein. At $24 \mathrm{~h}$ posttransfection, cells were harvested for luciferase assay (top panels) and western blotting (bottom panels). IRF3 proteins were tagged with HA while MERS-CoV proteins were FLAG-tagged. 
significant down-regulation of MDA5 protein levels by ORF8b (Fig. 1A, bottom panels and previous studies [22, 46]). Therefore, it is reasonable to propose that ORF8b may interact with CARD domains of RIG-I, sequestering them from interacting with the CARD domain of MAVS. As activation of RLRs by the recognition of the RNA genomes of invading pathogens ultimately lead to phosphorylation and activation of IRF3, the status of IRF3 phosphorylation was probed by phosphor-specific antibodies. As expected, down-regulation of MDA5 protein levels by ORF8b led to lower levels of phospho-IRF3 at the Ser386/396 residues (Fig. 1A, bottom panels). On the contrary, total levels of RIG-I were not perturbed by ORF8b (Fig. 1B, bottom panels) although ORF8b significantly inhibited RIG-Imediated activation of the IFN- $\beta$ promoter activity (Fig. 1B, upper panels). Interestingly, ORF8b and RIG-I coexpression led to lower levels of phosphor-IRF3 with albeit comparable levels of total endogenous IRF3 (Fig. 1B, bottom panels). These data suggest that ORF8b may inhibit CARD-CARD interactions between RIG-I and MAVS, leading to lower levels of IRF3 phosphorylation. Interestingly, none of the IRF3 variants (full-length (FL), IRF3-1-390 and IRF-5D) seemed to be affected by ORF8b (Fig. 4), suggesting that ORF8b may not directly inhibit IRF3 activation.

Taken together, here we report that ORF8b, encoded by MERS-CoV, is a strong antagonist of the RLR signaling pathway. ORF8b significantly down-regulated both MDA5and RIG-I-mediated induction of IFN- $\beta$ promoter activities. Interestingly, ORF8b inhibited all forms of RIG-I (fulllength, RIG-I-1-734 and RIG-I-1-228), implying that it may inhibit CARD-CARD interactions between RIG-I and MAVS without affecting protein levels of RIG-I, leading to lower levels of IRF3 phosphorylation. It is currently being investigated whether ORF8b directly interacts with the CARD domain of RLRs and/or MAVS. Delineation of the exact mechanisms of ORF8b-mediated antagonism of the RLR signaling pathway will pave way to development of effective virus-specific therapeutics.

\section{Acknowledgement}

This research was supported by HI15C3039 through the Korea Health Industry Development Institute (KHIDI) funded by the Korean Ministry of Health and Welfare and Basic Science Research Program through the National Research Foundation (NRF) funded by the Ministry of Education (2017R1A6A1A03015876).

\section{Conflict of Interest}

The authors have no financial conflicts of interest to declare.

\section{Reference}

1. Zaki AM, van Boheemen S, Bestebroer TM, Osterhaus AD, Fouchier RA. 2012. Isolation of a novel coronavirus from a man with pneumonia in Saudi Arabia. N. Engl. J. Med. 367: 1814-1820.

2. Corman VM, Eckerle I, Bleicker T, Zaki A, Landt O, Eschbach-Bludau M, et al. 2012. Detection of a novel human coronavirus by real-time reverse-transcription polymerase chain reaction. Euro Surveill. 17(39). pii: 20285.

3. Chan JF, Lau SK, To KK, Cheng VC, Woo PC, Yuen KY. 2015. Middle East respiratory syndrome coronavirus: another zoonotic betacoronavirus causing SARS-like disease. Clin. Microbiol. Rev. 28: 465-522.

4. Lee J, Bae S, Myoung J. 2019. Generation of full-length infectious cDNA clones of middle east respiratory syndrome coronavirus. J. Microbiol. Biotechnol. 29: 999-1007.

5. van den Brand JM, Smits SL, Haagmans BL. 2015. Pathogenesis of Middle East respiratory syndrome coronavirus. J. Pathol. 235: $175-184$.

6. Widagdo W, Sooksawasdi Na Ayudhya S, Hundie GB, Haagmans BL. 2019. Host Determinants of MERS-CoV Transmission and Pathogenesis. Viruses 11(3). pii: E280.

7. Mubarak A, Alturaiki W, Hemida MG. 2019. Middle East Respiratory Syndrome Coronavirus (MERS-CoV): Infection, Immunological Response, and Vaccine Development. J. Immunol. Res. 2019: 6491738.

8. Azhar EI, El-Kafrawy SA, Farraj SA, Hassan AM, Al-Saeed MS, Hashem AM, et al. 2014. Evidence for camel-to-human transmission of MERS coronavirus. N. Engl. J. Med. 370: 2499-2505.

9. Ferguson NM, Van Kerkhove MD. 2014. Identification of MERS-CoV in dromedary camels. Lancet Infect. Dis. 14: 93-94.

10. Haagmans BL, Al Dhahiry SH, Reusken CB, Raj VS, Galiano M, Myers R, et al. 2014. Middle East respiratory syndrome coronavirus in dromedary camels: an outbreak investigation. Lancet Infect. Dis. 14: 140-145.

11. Anthony SJ, Gilardi K, Menachery VD, Goldstein T, Ssebide B, Mbabazi R, et al. 2017. Further Evidence for Bats as the Evolutionary Source of Middle East Respiratory Syndrome Coronavirus. MBio. 8(2). pii: e00373-17.

12. Anthony SJ, Johnson CK, Greig DJ, Kramer S, Che X, Wells $\mathrm{H}$, et al. 2017. Global patterns in coronavirus diversity. Virus Evol. 3: vex012.

13. Banerjee A, Falzarano D, Rapin N, Lew J, Misra V. 2019. Interferon Regulatory Factor 3-Mediated Signaling Limits Middle-East Respiratory Syndrome (MERS) Coronavirus 
Propagation in Cells from an Insectivorous Bat. Viruses 11(2). pii: E152.

14. Goldstein SA, Weiss SR. 2017. Origins and pathogenesis of Middle East respiratory syndrome-associated coronavirus: recent advances. F1000Res. 6: 1628.

15. Hu B, Zeng LP, Yang XL, Ge XY, Zhang W, Li B, et al. 2017. Discovery of a rich gene pool of bat SARS-related coronaviruses provides new insights into the origin of SARS coronavirus. PLoS Pathog. 13: e1006698.

16. Li W, Shi Z, Yu M, Ren W, Smith C, Epstein JH, et al. 2005. Bats are natural reservoirs of SARS-like coronaviruses. Science 310: 676-679.

17. Yu P, Hu B, Shi ZL, Cui J. 2019. Geographical structure of bat SARS-related coronaviruses. Infect. Genet. Evol. 69: 224-229.

18. Zhou P, Fan $\mathrm{H}$, Lan T, Yang XL, Shi WF, Zhang W, et al. 2018. Fatal swine acute diarrhoea syndrome caused by an HKU2-related coronavirus of bat origin. Nature 556: 255-258.

19. Huang YW, Dickerman AW, Pineyro P, Li L, Fang L, Kiehne $\mathrm{R}$, et al. 2013. Origin, evolution, and genotyping of emergent porcine epidemic diarrhea virus strains in the United States. MBio. 4: e00737-00713.

20. Hayman DT. 2016. Bats as Viral Reservoirs. Annu. Rev. Virol. 3: 77-99.

21. Luis AD, Hayman DT, O'Shea TJ, Cryan PM, Gilbert AT, Pulliam JR, et al. 2013. A comparison of bats and rodents as reservoirs of zoonotic viruses: are bats special? Proc. Biol. Sci. 280: 20122753.

22. Lee JY, Bae S, Myoung J. 2019. Middle East respiratory syndrome coronavirus-encoded accessory proteins impair MDA5-and TBK1-mediated activation of NF-kappaB. J. Microbiol. Biotechnol. 29: 1316-1323.

23. Kang S, Myoung J. 2017. Host innate immunity against hepatitis $\mathrm{E}$ virus and viral evasion mechanisms. J. Microbiol. Biotechnol. 27: 1727-1735.

24. Theofilopoulos AN, Baccala R, Beutler B, Kono DH. 2005. Type I interferons (alpha/beta) in immunity and autoimmunity. Annu. Rev. Immunol. 23: 307-336.

25. Xi Y, Day SL, Jackson RJ, Ranasinghe C. 2012. Role of novel type I interferon epsilon in viral infection and mucosal immunity. Mucosal Immunol. 5: 610-622.

26. Zitvogel L, Galluzzi L, Kepp O, Smyth MJ, Kroemer G. 2015. Type I interferons in anticancer immunity. Nat. Rev. Immunol. 15: 405-414.

27. Kang S, Myoung J. 2017. Primary lymphocyte infection models for KSHV and its putative tumorigenesis mechanisms in B cell lymphomas. J. Microbiol. 55: 319-329.

28. Akira S, Uematsu S, Takeuchi O. 2006. Pathogen recognition and innate immunity. Cell 124: 783-801.

29. Fujita T, Onoguchi K, Onomoto K, Hirai R, Yoneyama M. 2007. Triggering antiviral response by RIG-I-related RNA helicases. Biochimie 89: 754-760.

30. Medzhitov R. 2007. Recognition of microorganisms and activation of the immune response. Nature 449: 819-826.
31. Kawai T, Takahashi K, Sato S, Coban C, Kumar H, Kato H, et al. 2005. IPS-1, an adaptor triggering RIG-I- and Mda5mediated type I interferon induction. Nat. Immunol. 6: 981988.

32. Meylan E, Curran J, Hofmann K, Moradpour D, Binder M, Bartenschlager R, et al. 2005. Cardif is an adaptor protein in the RIG-I antiviral pathway and is targeted by hepatitis C virus. Nature 437: 1167-1172.

33. Seth RB, Sun L, Ea CK, Chen ZJ. 2005. Identification and characterization of MAVS, a mitochondrial antiviral signaling protein that activates NF-kappaB and IRF 3. Cell 122: 669-682.

34. Kang S, Choi C, Choi I, Han KN, Rho SW, Choi J, et al. 2018. Hepatitis E virus methyltransferase inhibits type I interferon induction by targeting RIG-I. J. Microbiol. Biotechnol. 28: 1554-1562.

35. Myoung J, Min K. 2019. Dose-dependent inhibition of melanoma differentiation-associated gene 5-mediated activation of type I interferon responses by methyltransferase of hepatitis E virus. J. Microbiol. Biotechnol. 29: 1137-1143.

36. Chau TL, Gioia R, Gatot JS, Patrascu F, Carpentier I, Chapelle JP, et al. 2008. Are the IKKs and IKK-related kinases TBK1 and IKK-epsilon similarly activated? Trends Biochem. Sci. 33: 171-180.

37. Clement JF, Meloche S, Servant MJ. 2008. The IKK-related kinases: from innate immunity to oncogenesis. Cell Res. 18: 889-899.

38. Fitzgerald KA, McWhirter SM, Faia KL, Rowe DC, Latz E, Golenbock DT, et al. 2003. IKKepsilon and TBK1 are essential components of the IRF3 signaling pathway. Nat. Immunol. 4: 491-496.

39. Gatot JS, Gioia R, Chau TL, Patrascu F, Warnier M, Close P, et al. 2007. Lipopolysaccharide-mediated interferon regulatory factor activation involves TBK1-IKKepsilon-dependent Lys(63)-linked polyubiquitination and phosphorylation of TANK/I-TRAF. J. Biol. Chem. 282: 31131-31146.

40. Hacker H, Karin M. 2006. Regulation and function of IKK and IKK-related kinases. Sci. STKE. 2006: re13.

41. Grandvaux N, Servant MJ, tenOever B, Sen GC, Balachandran S, Barber GN, et al. 2002. Transcriptional profiling of interferon regulatory factor 3 target genes: direct involvement in the regulation of interferon-stimulated genes. J. Virol. 76: 5532-5539.

42. Honda K, Taniguchi T. 2006. IRFs: master regulators of signalling by Toll-like receptors and cytosolic patternrecognition receptors. Nat. Rev. Immunol. 6: 644-658.

43. Liu S, Cai X, Wu J, Cong Q, Chen X, Li T, et al. 2015. Phosphorylation of innate immune adaptor proteins MAVS, STING, and TRIF induces IRF3 activation. Science 347: aaa2630.

44. Hiscott J, Grandvaux N, Sharma S, Tenoever BR, Servant MJ, Lin R. 2003. Convergence of the NF-kappaB and interferon signaling pathways in the regulation of antiviral defense and apoptosis. Ann. NY Acad. Sci. 1010: 237-248. 
45. Wang J, Basagoudanavar SH, Wang X, Hopewell E, Albrecht R, Garcia-Sastre A, et al. 2010. NF-kappa B RelA subunit is crucial for early IFN-beta expression and resistance to RNA virus replication. J. Immunol. 185: 1720-1729.

46. Lee JY, Bae S, Myoung J. 2019. Middle East respiratory syndrome coronavirus-encoded ORF8b strongly antagonizes IFN-beta promoter activation: its implication for vaccine design. J. Microbiol. 57: 803-811.

47. Hu J, Lou DH, Carow B, Winerdal ME, Rottenberg M, Wikstrom AC, et al. 2012. LPS regulates SOCS2 transcription in a Type I Interferon dependent autocrine-paracrine loop. PLoS One 7(1): e30166.

48. Song JW, Guan M, Zhao ZW, Zhang JJ. 2015. Type I interferons function as autocrine and paracrine factors to induce autotaxin in response to TLR activation. PLoS One 10(8): e0136629.

49. Hasan MT, Jang WJ, Tak JY, Lee BJ, Kim KW, Hur SW, et al. 2018. Effects of Lactococcus lactis subsp. lactis I2 with betaGlucooligosaccharides on growth, innate immunity and Streptococcosis resistance in olive flounder (Paralichthys olivaceus). J. Microbiol. Biotechnol. 28: 1433-1442.

50. Liu S, Yu X, Wang Q, Liu Z, Xiao Q, Hou P, et al. 2017. Specific expression of interferon-gamma induced by synergistic activation mediator-derived systems activates innate immunity and inhibits Tumorigenesis. J. Microbiol. Biotechnol. 27: 1855-1866.
51. Baek YH, Cheon HS, Park SJ, Lloren KKS, Ahn SJ, Jeong JH, et al. 2018. Simple, rapid and sensitive portable molecular diagnosis of SFTS virus using reverse transcriptional loopmediated isothermal amplification (RT-LAMP). J. Microbiol. Biotechnol. 28: 1928-1936.

52. Jo G, Jeong MS, Wi J, Kim DH, Kim S, Kim D, et al. 2018. Generation and characterization of a neutralizing human monoclonal antibody to Hepatitis B Virus PreS1 from a Phage-Displayed human synthetic fab library. J. Microbiol. Biotechnol. 28: 1376-1383.

53. Kim E, Myoung J. 2018. Hepatitis E Virus papain-like cysteine protease inhibits type I interferon induction by down-regulating melanoma differentiation-associated gene 5 . J. Microbiol. Biotechnol. 28: 1908-1915.

54. Park MK, Cho H, Roh SW, Kim SJ, Myoung J. 2019. Cell type-specific interferon-gamma-mediated antagonism of KSHV lytic replication. Sci. Rep. 9: 2372.

55. Ngueyen TTN, Kim SJ, Lee JY, Myoung J. 2019. Zika virus proteins NS2A and NS4A are major antagonists that reduce IFN-beta promoter activity induced by the MDA5/RIG-I signaling pathway. J. Microbiol. Biotechnol. 29: 1665-1674.

56. Loo YM, Fornek J, Crochet N, Bajwa G, Perwitasari O, Martinez-Sobrido L, et al. 2008. Distinct RIG-I and MDA5 signaling by RNA viruses in innate immunity. J. Virol. 82: 335-345.

57. Takeuchi O, Akira S. 2010. Pattern recognition receptors and inflammation. Cell 140: 805-820. 